\title{
Antibodies to HLA Molecules Mimic Agonistic Stimulation to Trigger Vascular Cell Changes and Induce Allograft Injury
}

\author{
Nicole M. Valenzuela ${ }^{1} \cdot{\text { Elaine F. } \text { Reed }^{1}}^{1}$
}

Published online: 24 May 2015

(C) Springer International Publishing AG 2015

\begin{abstract}
Human leukocyte antigen (HLA)-induced signaling in endothelial and smooth muscle cells causes dramatic cytoskeletal rearrangement, increased survival, motility, proliferation, adhesion molecule and chemokine expression, and adhesion of leukocytes. These mechanisms are directly related to endothelial activation, neointimal proliferation, and intragraft accumulation of leukocytes during antibody-mediated rejection (AMR) and chronic rejection. Clustering of HLA by ligands in trans, such as in antigen-presenting cells at the immune synapse, triggers physiological functions analogous to HLA antibody-induced signaling in vascular cells. Emerging evidence has revealed previously unknown functions for HLA beyond antigen presentation, including association with coreceptors in cis to permit signal transduction, and modulation of intracellular signaling downstream of other receptors that may be relevant to HLA signaling in the graft vasculature. We discuss the literature regarding HLA-induced signaling in vascular endothelial and smooth muscle cells, as well as under endogenous biological conditions, and how such signaling relates to functional changes and pathological mechanisms during graft injury.
\end{abstract}

This article is part of the Topical Collection on Immunology

Elaine F. Reed

ereed@mednet.ucla.edu

Nicole M. Valenzuela

npyburn@mednet.ucla.edu

1 UCLA Immunogenetics Center, Department of Pathology and Laboratory Medicine, David Geffen School of Medicine, University of California, 1000 Veteran Ave Room 1-520, Los Angeles, CA 90095, USA
Keywords HLA antibodies · Endothelial cells · Antibody-mediated rejection $\cdot$ Leukocyte recruitment $\cdot$ Signal transduction $\cdot$ Mammalian target of rapamycin (mTOR)

\section{Introduction}

Antibodies against donor human leukocyte antigen (HLA) molecules and subsequent rejection episodes are strongly associated with risk of chronic rejection and late graft failure [1-3]. The general mechanisms of graft injury by HLA antibodies during antibody-mediated rejection (AMR) include endothelial injury and dysfunction, infiltration of innate immune cells, and activation of the classical complement cascade. While canonical antibody Fc-mediated activation of complement- and Fc $\gamma$ R-dependent functions are undoubtedly important for the pathogenesis of antibody-mediated rejection, agonistic actions of HLA antibodies on donor vascular cells cause functional and phenotypic changes leading to endothelial and smooth muscle cell dysfunction that contribute to graft injury and rejection. This review will summarize and discuss the current knowledge about HLA-induced signaling in vascular cells and how it relates to graft injury during rejection. In addition, we will attempt to address how and why HLA molecules induce intracellular signaling upon cross-linking and what can be inferred from the known physiological roles of HLA in the immune system and beyond.

Although this review focuses on the mechanisms of HLAmediated signaling within vascular cells, which contribute to graft pathology and injury, it is important to keep in mind that antibody-mediated rejection is a multifaceted process that involves other mechanisms of injury. We and others have proposed that the concomitant effects of antibodies, $F\left(a b^{\prime}\right)_{2}$-dependent endothelial activation, $\mathrm{Fc} \gamma \mathrm{R}$-dependent leukocyte functions [4, 5], and $\mathrm{Fc}$-dependent activation of complement 
[6••], synergize during antibody-mediated rejection to culminate in graft injury. Thus, parallel mechanisms of complement activation and $\mathrm{Fc} \gamma \mathrm{R}$ functions likely exacerbate endothelial dysfunction and graft injury initiated by HLA signaling.

\section{HLA Signaling in Vascular Cells in Response to Antibodies: Mechanisms Related to Graft Injury}

It has been well established that agonistic cross-linking of HLA I by alloantibodies activates a phosphorylationdependent signaling cascade in vascular cells that leads to functional changes that may contribute to the histological manifestations of graft injury during acute and chronic rejection (Fig. 1a). In contrast, study of HLA II in cultured vascular cells has been technically challenging. Recently, two approaches to circumvent this complication have been described $[7,8 \bullet]$, which will likely permit much-needed studies of HLA II antibody effects on endothelium in the near future. For the purposes of this review, we will indicate, where possible, any existing knowledge regarding HLA II in vascular cells or any relevant literature from other cell types, but the reader should recognize that the evidence is currently limited.

\section{Cytoskeletal Reorganization: Migration, Permeability, and Signal Localization}

One of the earliest functional changes occurring after exposure of endothelial cells to HLA I antibodies is the dynamic remodeling of the actin cytoskeleton, resulting in rapid and dramatic stress fiber formation [9-11, 12•]. Rho GTPase is activated after HLA I ligation by antibodies [10, 13]. Src protein kinase is rapidly activated and subsequently phosphorylates focal adhesion kinase (FAK) in endothelium [14] and smooth muscle cells [15]. FAK targets paxillin, an adaptor found in focal adhesion which transmits adhesion-dependent intracellular signaling. Although the role of HLA II in regulating endothelial cytoskeleton has not yet been reported, FAK was activated in fibroblasts after stimulation with HLA II antibodies [16]. Rho and Rho kinase were critically required for HLA I-induced FAK and paxillin phosphorylation as well as stress fiber formation $[10,11]$. Other GTPases, Rac and Cdc42, which also regulate actin cytoskeletal organization, were unchanged in endothelium after HLA I cross-linking [10]. This finding suggests that the type of stress fiber formed after HLA I cross-linking is distinct from Rac- and Cdc42associated lamellipodia and filopodia and rather results in the formation of focal adhesion [17]. This is consistent with the observation that the pattern of stress fibers is different from those stimulated by thrombin [11]; thrombin acts on both Rho and Rac to control the actin cytoskeleton [18], which have opposing actions on the cytoskeleton in endothelium.
We also reported that ERK1/2 phosphorylation was dependent on mammalian target of rapamycin (mTOR) complex 2 (mTORC2) following HLA I ligation [19]. ERK proteins are vital for growth factor and integrin signaling and also regulate the cytoskeleton. Ultimately, Rho and ERK converge to activate myosin light chain (MLC) [11], which plays a central role in actin contractility and stress fiber assembly. Notably, activation of ERK through mTORC2 was unique to HLA I and integrin-induced signaling but was not observed with growthfactor-induced signaling [19], pointing to a distinct pathway of ERK activation that is common to MHC class I and integrins. Subsequent studies revealed that both mTORC2 and ERK were required for HLA I-mediated stress fiber formation in endothelium [11] and that pharmacological inhibition of mTOR with rapalogs could prevent stress fiber formation after exposure to HLA I antibodies [19].

What is the purpose or functional outcome of actin stress fiber formation? The cytoskeleton is integral to cell motility, cytokinesis, endocytic and exocytic events, cell-cell interactions, and invasion, as well as facilitating inside-out signaling of transmembrane receptors and integrins [20,21]. The function of stress fibers is context dependent on their intracellular location, morphology, and associated proteins [22]. Stress fiber connection to the extracellular matrix through focal adhesion, such as those observed after HLA I antibody activation in endothelial cells, permits mechanotransduction. Stress fibers of this type are also likely important for endothelial rigidity during tethering of leukocytes [23] and during formation of cell-cell synapses.

It is increasingly appreciated that the cytoskeleton is also an active organizer of intracellular signaling cascades, controlling localization and compartmentalization of discrete processes and pathways. Indeed, disruption of the endothelial cytoskeleton abolishes all downstream signaling [14] and prevents molecular aggregation of HLA I by antibodies. Consistent with its central role in coordinating signaling molecules after HLA I cross-linking by antibodies in endothelial cells, the repertoire of phosphorylated signaling proteins associated with the actin cytoskeleton was dramatically altered [12•]. Using mass spectrometric analysis, Ziegler et al. verified that proteins involved in catalytic functions, protein biosynthesis, and actin cytoskeletal organization were dynamically associated with the cytoskeletal fraction in response to HLA ligation [12•]. mTORC2 and ERK were shown to translocate to the plasma membrane from the cytoplasm, where they may colocalize with transmembrane receptors and lipid rafts to facilitate signaling [11]. HLA I cross-linking by antibodies also resulted in increased endothelial and smooth muscle cell motility in in vitro wound-healing models of cell migration, a process which is important for angiogenic functions [24•].

Therefore, HLA I induction of cytoskeletal reorganization is a critical step in coordinating intracellular signaling pathways and downstream functional changes in vascular 


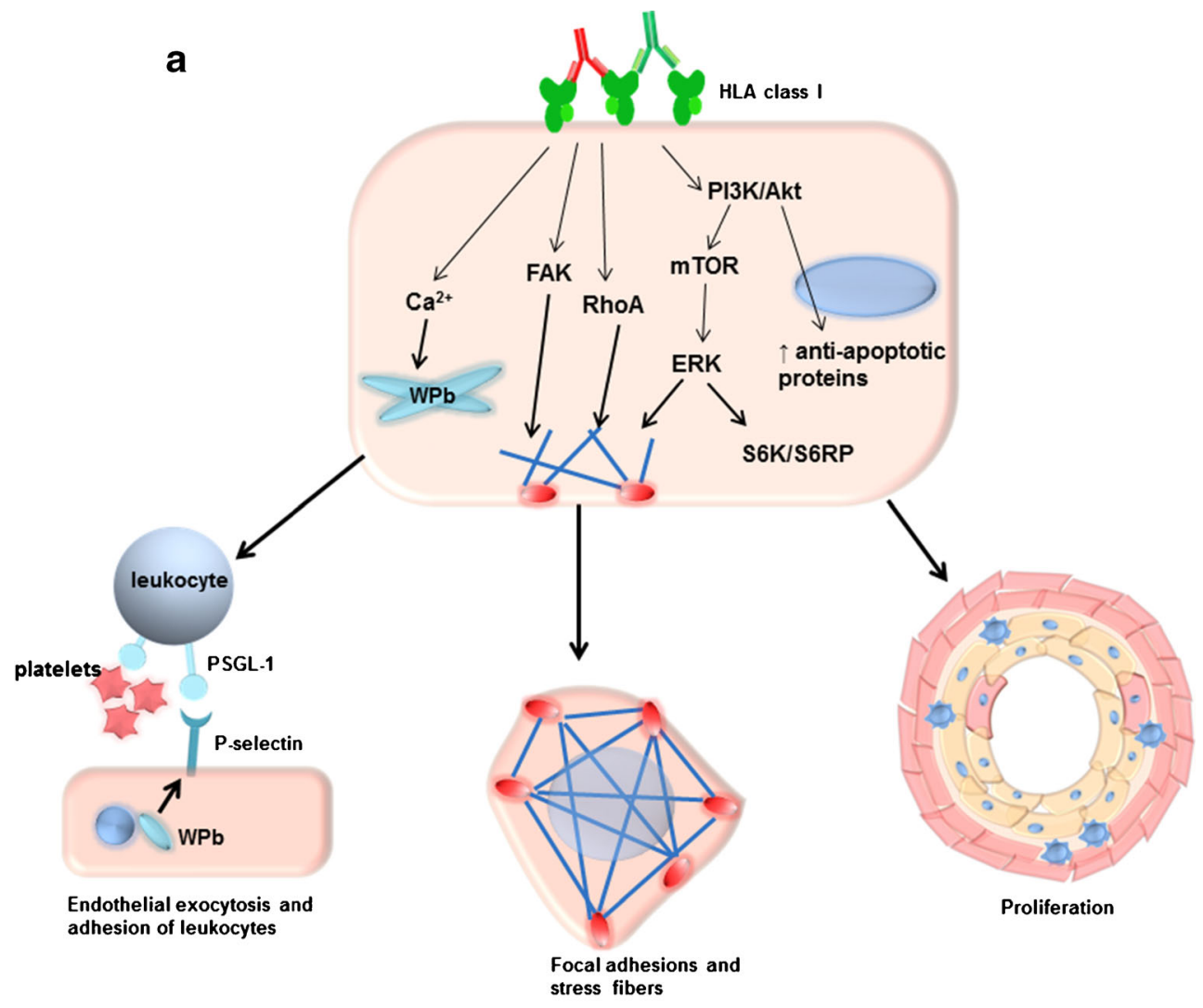

b

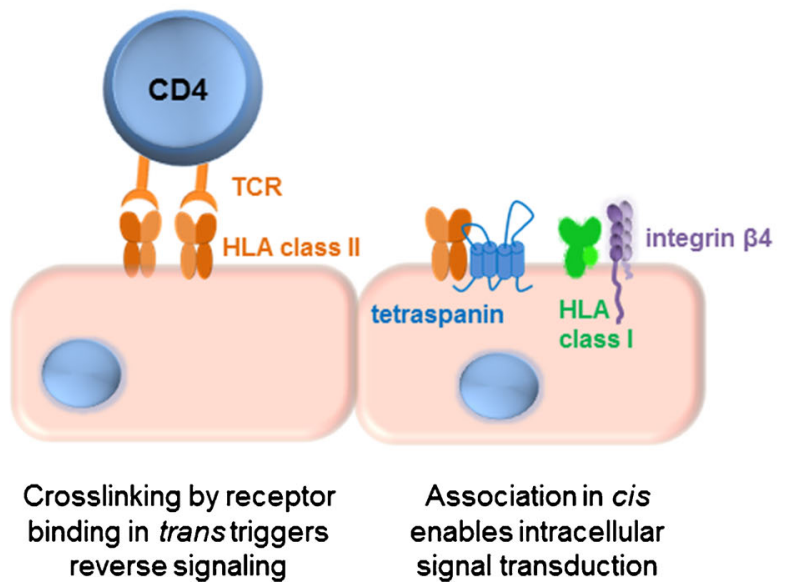

Fig. 1 a Cross-linking of HLA I on the surface of endothelial and smooth muscle cells activates intracellular signaling programs that promote the mechanisms of graft injury. Intracellular calcium is released in endothelium, which triggers exocytosis of Weibel-Palade body $(\mathrm{WPb})$ vesicles containing P-selectin. Rapid upregulation of P-selectin at the cell surface is sufficient to support adhesion of neutrophils, monocytes, and platelets, which likely contributes to the predominance of intragraft myeloid cells, such as CD68+ macrophages and neutrophils, during antibody-mediated rejection. HLA signaling also promotes activation of FAK, which facilitates formation of focal adhesions, and activation of RhoA, mTOR, and ERK, which act on the actin cytoskeleton to stimulate stress fibers. Stress fiber formation is dramatic and extends across the cell. mTOR and ERK are also key regulators of proteins involved in translation, S6 kinase

(S6K) and $\mathrm{S} 6$ ribosomal protein (S6RP), and of cell growth. Activation of the PI3K/Akt axis causes upregulation of pro-survival proteins. Together, proliferative, survival, and migratory signaling likely contributes to chronic vascular rejection and intimal hyperplasia. b Schematic diagram of HLA binding in trans to receptors on opposing cells, such as the TCR on T cells, and of HLA association in cis with tetraspanins and integrins on the same cell. Trans interactions are likely the physiologically relevant mechanisms of HLA cross-linking that leads to reverse signaling into the cell, pathways which become hijacked and inappropriately activated by bivalent alloantibody during antibody-mediated rejection. Associations in cis are required for HLA, which has no known signaling motifs, to transduce signaling through coreceptors, such as integrin $\beta 4$ in endothelial cells 
endothelial and smooth muscle cells. In addition, dynamic regulation of the actin cytoskeleton following HLA antibody binding might account for the microvascular endothelial changes observed by electron microscopy in renal allograft AMR. However, it should be noted that antagonism of endothelial cytoskeletal functions may also have detrimental effects on wound healing, as has been observed in patients receiving mTOR inhibitors such as rapamycin.

\section{Survival: Endothelial Resistance to Injury and Persistence}

Survival of donor vascular cells despite ongoing graft injury, in particular complement-mediated inflammation, is thought to be an important contributor to chronic rejection. Low concentrations of HLA antibodies induce a cytoprotective program in endothelial cells that prevents lysis upon exposure to high doses of HLA antibody. HLA cross-linking activated the PI3K/Akt signaling axis at low antibody concentrations of antibody, causing upregulation of heme oxygenase-1 (HO-1) and ferritin [25, 26]. Additionally, PI3K/Akt upregulated the anti-apoptotic proteins Bcl-2 and Bcl-xL, as well as induced phosphorylation of Bcl-2-associated death promoter (Bad) [27]. These effects negatively regulate mitochondrial-controlled cell death, by attenuating apoptosis and antagonizing the cytotoxic effects of complement. HLA class II also appears to regulate endothelial cell signaling. Cross-linking of HLA II on IFN-activated endothelium activates intracellular signaling preventing apoptosis [28], which the authors noted was a distinct response of endothelium compared with B cells.

Recent work indicates that accommodation of allografts in the presence of HLA antibodies may not be an indefinite state, however. Several studies have uncovered "indolent," "subclinical," or "asymptomatic" inflammatory [29-32] markers in grafts of patients with digital subtraction angiography (DSA) but without current dysfunction. The "natural history of antibodies" proposed by Wiebe et al. [33・•] suggests that ongoing inflammation in the graft occurs early after DSA appears, although histological and clinical presentation of graft rejection does not manifest until later. Therefore, the prosurvival signaling cascades initiated by low titers of HLA antibodies may protect endothelial cells from primary injury or death by complement-induced lysis but permit donor endothelial cell persistence that sets the stage for cell growth observed during chronic rejection, described in the next section.

\section{Proliferation: Intimal Expansion and Angiogenesis}

Antibodies are significantly associated with chronic rejection and late allograft loss in many solid organs [32, 34]. Chronic rejection manifests generally as perivascular fibrosis and expansion of the intimal layer of the donor vasculature. For example, in transplant vasculopathy of cardiac allografts, donor coronary arteries become occluded, with a hypertrophic endothelial layer invaded by smooth muscle cells, resulting in narrowing of the lumen [35]. Chronic rejection in kidney transplants, transplant glomerulopathy, appears as duplication of the basement membrane and interstitial fibrosis with obliteration of the glomerular and peritubular capillary lumens [36]. In lung allografts, bronchiolitis obliterans results in fibrosis affecting the small airways; pulmonary vascular rejection may also be present [37, 38]. Angiogenic processes appear to be central to formation of vascular lesions in chronic rejection, where vascular remodeling results in new smoothmuscle-lined microvessels [39-42].

In the absence of inflammation or injury, endothelial cells are quiescent and cell growth is static. Chronic inflammation and injury trigger endothelial cell turnover and repair mechanisms. Ample evidence has shown that cross-linking of MHC class I on endothelial and smooth muscle cells triggers increased proliferation in vitro $[9,15,43,44]$ and in vivo $[45$, 46]. Cell growth is dependent upon proximal activation of RhoA [13], mTOR, S6 kinase and S6 ribosomal protein (S6RP), and ERK [19, 24•]. Activation of these signaling molecules can be readily detected in murine cardiac allografts exposed to anti-donor MHC class I antibodies [43] as well as in cardiac biopsies from patients with donor-specific HLA antibodies [47, 48, 49॰]. Indeed, phosphorylated S6RP is a useful marker of ongoing AMR. Pharmacological antagonism of mTOR using the rapalogs sirolimus and everolimus significantly attenuated HLA I antibody-induced endothelial proliferation in vitro [24•]. Interestingly, the capacity of everolimus but not sirolimus to inhibit mTORC2 and ERK1/2 was essential for full prevention of endothelial proliferation. Recently, a novel endogenous regulator of mTOR, called DEPTOR, was described in endothelial cells and may represent a therapeutic target to modulate HLA antibody-induced proliferation [50 ${ }^{\bullet}$. These results suggest that mTOR inhibitors, particularly those that effectively block mTORC2, may have utility in preventing chronic AMR. Indeed, patients treated with everolimus experienced a slower progression of chronic cardiac allograft rejection (CAV) compared with standard MMFbased immunosuppression [51, 52].

A central stimulator of angiogenic responses is vascular endothelial cell growth factor (VEGF), which is upregulated in the circulation and in graft endothelial cells of patients with vascular lesions [53, 54]. VEGF was critical for neovascularization and neointimal formation in a rat model of chronic rejection [55]. Endothelial cells stimulated with HLA I antibodies upregulate VEGF [56], which may act in an autocrine fashion to enhance endothelial growth. Furthermore, inside-out signaling in endothelium after stimulation with VEGF causes increased vascular permeability through FAK and VE-cadherin, which may allow paracellular leakage of proteins, including immunoglobulin IgG. It is plausible that changes in endothelial permeability permit access of HLA antibodies to the subendothelial space, where they may bind to smooth muscle cells. 
Smooth muscle cells contribute to transplant vasculopathy by invading and proliferating in the subendothelial and intimal layer [39]. Recent work demonstrated that HLA I signaling in smooth muscle cells promotes mitogenesis and migration through FAK, Akt, and ERK/12 [15], as well as increased activity of matrix metalloproteinase (MMP) and sphingomyelinases, leading to intimal expansion in a murine xenograft model of human arterial segments [57]. Pharmacological inhibitors of MMPs reduced HLA antibody-associated intimal expansion in vivo, implicating, but not conclusively defining, a role for autocrine signaling by the sphingolipid sphingosine-1-phosphate (S1P) in this process.

\section{Inflammation and Alloimmunogenicity}

Accumulation of myeloid cells within the allograft is an important histological marker of antibody-mediated rejection, and macrophages populate the vascular lesions in chronic rejection [39]. In lung allografts, neutrophilic capillaritis is associated with circulating donor-specific antibodies [3], while the macrophage marker CD68 is central to the diagnosis of antibody-mediated rejection of cardiac allografts [58], and macrophage burden predicts poorer outcome in cardiac and renal transplantation $[59,60]$. In addition, molecular diagnostic studies of antibody-mediated rejection of renal allografts have revealed NK cell- and macrophage-associated signatures [29]. NK cell and macrophage accumulation in allografts has been recapitulated in murine models of AMR [45, 61-63].

Endothelial cells are active modulators of the immune response. At the interface between the donor tissue and the recipient immune system, they coordinate recruitment of leukocytes from the blood into sites of inflammation. Endothelial cells store vasoactive mediators, chemokines, and adhesion molecules in intracellular vesicles called Weibel-Palade bodies $(\mathrm{WPb})$ which are rapidly mobilized during type I endothelial activation [64]. Two notable constituents of $\mathrm{WPb}$ are von Willebrand factor (vWF), a regulator of clotting, and Pselectin, an initiator of leukocyte tethering to endothelium. Yamakuchi et al. first demonstrated the rapid increase in cell surface P-selectin in response to HLA I cross-linking, which supported increased neutrophil adherence in vitro and in vivo [65]. vWF was also rapidly released into the extracellular space after HLA antibody-induced exocytosis. Calciumdependent exocytic pathways involving the ATPase $N$ ethylmaleimide sensitive factor (NSF) were critical for this response [65]. vWF staining could be observed in vessels of grafts treated with anti-donor MHC antibody but was lost 7 days after exposure to antibody, suggesting that this process is transient [66]. Monocytes were also recruited through endothelial P-selectin, and antagonism of P-selectin abrogated infiltration of macrophages into murine cardiac allografts [62]. Both $\mathrm{vWF}$ and $\mathrm{P}$-selectin can promote platelet aggregation in the microvasculature, and anti-donor MHC class I antibodies induced rolling of platelets in the vessels of an allogeneic skin graft [67]. In addition to their central role in thrombosis, platelets can potently bridge leukocyte tethering to the vessel wall, and in vivo, this may be an important mechanism of efficient monocyte recruitment [68].

There is also evidence that HLA cross-linking causes late phase endothelial cell activation. Stimulation of microvascular endothelial cells with HLA antibodies caused activation of the transcription factors, with subsequent upregulation of cytokines and chemokines $[6 \bullet \bullet, 69,70]$. Evidence from work with IFN $\gamma$-activated fibroblasts suggests that HLA II cross-linking also upregulates inflammatory factors [71]; but this has not been clearly defined in endothelial or smooth muscle cells. Abe et al. demonstrated in a murine model of AMR that allografts deficient in the chemokine monocyte chemoattractant protein-1 (MCP1/CCL2) had a reduction in intragraft macrophages [63], highlighting the importance of production of chemotactic factors by the graft during AMR. mTOR antagonism with rapamycin has been shown to inhibit endothelial activation, including adhesion molecule and chemokine production, by TNF $\alpha$ [72]. Therefore, it is possible that mTOR inhibition might prevent HLA antibody-induced transcriptional changes as well.

Emerging literature also supports the function of endothelial cells as semiprofessional antigen-presenting cells capable of stimulating T cell activation [73-75], a capacity that smooth muscle cells, epithelial cells, and fibroblasts lack [73, 76]. Activated endothelial cells (for example, with IFN $\gamma$ ) express, among many other factors, HLA II and are capable of activating $\mathrm{T}$ cells through CD58/CD2 interactions. Endothelium is sufficient to activate resting murine memory $\mathrm{T}$ cells but not naïve cells, due to a lack of B7.1 and B7.2 (CD80/CD86) expression $[74,77]$. Experimental evidence shows that HLA II-expressing endothelial cells trigger allogeneic CD4 T cell proliferation and promote generation of Th17 and Treg subsets $[7,8 \cdot$, 78]. Interestingly, rapamycin treatment of endothelial cells resulted in selective expansion of Tregs via PD-L1 and PD-L2 [8•], suggesting that mTOR regulates endothelial alloimmunogenicity. These novel $\mathrm{T}$ cell interactions are relevant to antibodymediated rejection, as the role of CD4 cells in so-called "mixed" AMR is increasingly reported in experimental models [79]. It has also been proposed that HLA antibodies may modulate endothelial immunogenicity $[6 \bullet \cdot]$, a fascinating avenue of investigation that remains to be fully illuminated.

\section{Why Does HLA Signal in Cells? Understanding the Role of Cross-linking by Trans Associations with Ligands}

Although the literature clearly demonstrates that ligation of HLA molecules by antibodies causes intracellular signaling and cell functional changes in vitro and in vivo, it is not 
generally appreciated in the transplant community why this occurs. Is it an unfortunate phenomenon idiosyncratic to humoral alloimmunity or hijacking of a real biological function of HLA signaling?

\section{Classical Roles of MHC in the Immune System}

The physiological role of classical HLA is presentation of antigen and self/non-self-recognition through binding in trans (i.e., on the plasma membrane of a different cell) to the $\mathrm{T}$ cell receptor (TCR) on T cells or to MHC ligands (e.g., killer cell immunoglobulin-like receptors, KIRs) on NK cells. It is readily apparent from studies of the immunologic synapse that engagement of TCR by MHC presenting cognate peptides causes clustering of MHC, with enrichment at the T cell- antigen-presenting cell (APC) interface [80, 81]. Therefore, trans association of MHC molecules with cognate receptors on other cells, such as in the case of biological interactions with the TCR, causes crosslinking of MHC analogous to alloantibody-mediated cross-linking (Fig. 1b).

Dynamic crosstalk at the immune synapse provides bidirectional signaling into both the T cell and the APC regulating APC function as well as lymphocyte activation and differentiation [82, 83]. Many studies have elucidated the "reverse signaling" of MHC (analogous to outside-in signaling) into the APC after engagement by TCRs or antibodies. Engagement of MHC class II causes actin polymerization to facilitate its enrichment at the immunologic synapse, relying on Rho/ Rac activation [84, 85]. MHC class II ligation also modulates survival of immature $\mathrm{T}$ cells during thymic development and of APCs and B cells $[28,86]$. Cross-linking of MHC class I and class II on T cells, B cells, monocytes, and dendritic cells causes activation of protein kinase $\mathrm{C}$ (PKC) and increased tyrosine phosphorylation and intracellular calcium. Cell outcomes are cell lineage- and context-dependent, including activation, proliferation, or apoptosis (reviewed elsewhere [86-93]). Thus, in its normal guise, reverse signaling by $\mathrm{MHC}$ is thought to be a mechanism of homeostatic regulation of the immune response, which acts on the cytoskeleton and protein tyrosine kinases, to regulate cell survival and growth.

\section{MHC Nonclassical Roles in the Immune System}

In addition to their role in antigen presentation of peptides and self to T cells and NK cells, HLA molecules carry out a variety of "nonclassical" functions within and outside of the immune system [93]. Recent work has shown that MHC class IIassociated invariant chain (CD74) negatively regulates macrophage and dendritic cell motility through actions on myosin II [94], demonstrating a coupling of MHC-dependent antigen presentation processes with cytoskeletal regulation. MHC class I reverse signaling also negatively regulates the function of NK cells after engagement by as yet unidentified ligands on target cells [95], and cross-linking of MHC class I inhibited cytotoxicity against tumor cells in response to CD16/Fc $\gamma$ RIII but not NKG2D stimulation [96•]. Therefore, HLA signaling in immune cells modulates cell function beyond the immunologic synapse.

\section{How Does HLA Transduce Intracellular Signals? Cis Associations}

The cytoplasmic tails of both HLA I and II are relatively short (30-40 amino acids) and lack any known signaling motif. However, the robust intracellular signaling and functional changes induced by engagement of HLA I and II strongly imply a cis association with other receptors capable of signal transduction (i.e., lateral interactions of HLA with other transmembrane receptors on the same cell) (Fig. 1b).

\section{Cis-Interacting Partners of MHC}

Many lines of evidence have demonstrated that the cytoplasmic domain of MHC is indispensable for transduction of signaling and association with the cytoskeleton after crosslinking [90, 97], including in endothelial cells [98]. Several serine residues and a tyrosine residue in the cytoplasmic domain of HLA I are known substrates for phosphorylation by Src family kinases and PKC [99-101], thought to be important for transport during peptide loading and endocytic recycling [102]. Phosphorylation of the HLA I intracellular domain leads to association with intracellular tyrosine kinases and subsequent HLA I-mediated signaling cascades in APCs [101]. It is possible that HLA also becomes phosphorylated in endothelial and smooth muscle cells after cross-linking, which would permit association with intracellular signaling adaptors, although this has not yet been demonstrated.

Given the similarities between HLA I-induced intracellular signaling and the signaling cascades downstream of integrins and growth factors, Zhang et al. hypothesized that HLA partnered with these receptors and discovered that HLA I associates with integrin $\beta 4$ at the cell surface of endothelial cells after cross-linking of HLA. This molecular association was dependent upon the cytoplasmic domain of HLA I and was required for antibody-induced phosphorylation of Src, Akt, and ERK, as well as cell migration and proliferation [98]. Whether HLA II associates with integrins or other receptors in endothelial cells has yet to be determined but may be inferred from observations in other cell types.

MHC molecules associate with a variety of tetraspanins in the endosomal compartment and at the plasma membrane in immune cells [103-107]. In addition, it has recently been elucidated that MHC class I ligands called leukocyte immunoglobulin-like receptors (LILRs/ILTs/CD85) interact with $\mathrm{MHC}$ molecules laterally, i.e., in the plane of the plasma 
membrane on the same cell as well as in trans (i.e., as ligands on opposing cells) [108]. Unlike TCRs and KIRs, LILRB proteins appear to have broad specificity for HLA I alleles, as the recognition site lies at the relatively lowly polymorphic $\alpha 3$ domain and the invariant $\beta 2$ microglobulin $[109,110]$. Notably, though, there was a range of affinities of LILRB1 for different alleles of HLA [111], and it is possible that interactions with LILRs vary among individuals with different HLA genotypes.

\section{MHC Role in Cis Regulation of Tonic Signaling in the Immune System and Beyond}

MHC molecules modulate intracellular signaling downstream of other receptors. For example, HLA I-LILR complexes forming in cis dampen activation of mast cells through $\mathrm{Fc}$ receptors [112]. Loss of MHC I and II in mice causes unexpected impairment of a variety of biological responses not previously thought to involve these molecules. For example, constitutive MHC class I played an integral role in dampening intracellular toll-like receptor (TLR) signaling in response to endotoxin, preventing sepsis [101]. In contrast, MHC class II was required for TLR signaling in macrophages [113], suggesting that MHC class I and MHC class II might differ in their intracellular signaling functions.

Recent work has elucidated the critical role for MHC in neuronal synapse elimination and pruning during development [114•], at least in the mouse. MHC associates in cis with insulin receptors on neurons, hepatic cells, and B cells, forming a molecular complex which modulates insulin receptor-dependent signaling and glucose uptake $[115,116]$. Complex formation of MHC class I with insulin receptor is thought to tonically inhibit insulin receptor signaling in neuronal pruning [117] and to downmodulate myeloma cell survival in the presence of low levels of insulin.

We also uncovered an unexpected requirement for HLA I in integrin $\beta 4$-mediated signaling. Surprisingly, endothelial cells lacking HLA I exhibited impaired integrin $\beta 4-$ dependent angiogenic functions, including intracellular signaling, proliferation, and migration [98]. Given the role of integrin $\beta 4$ in angiogenesis [118], HLA I molecules may act in concert with integrin $\beta 4$ to promote tumor progression [119]. These results add to the list of unforeseen functions of HLA in modulating intracellular signaling by other receptors and suggest that the association between HLA I and integrin $\beta 4$ is a physiological norm.

\section{Conclusions}

HLA molecules display diverse functions both within and without the immune system. Alloantibodies to HLA in the context of transplantation mimic the physiological clustering of HLA molecules, as at the immunologic synapse, to induce "reverse signaling" in donor vascular cells that is reminiscent of modulation of APC function and survival. Cross-linking of HLA by alloantibodies stimulates tyrosine kinase cascades leading to cytoskeletal rearrangement, endothelial resistance to complement-mediated lysis, and increased survival proteins, proliferation, and leukocyte recruitment and may modulate alloimmunogenicity to $\mathrm{T}$ cells. In addition, recent evidence points to novel roles for HLA molecules in the regulation of intracellular signaling downstream of many other cell surface receptors, including CD16/Fc $\gamma$ RIII in NK cells, integrin $\beta 4$ during angiogenic responses of endothelial cells, TLR-dependent responses in monocytes, and insulin receptor during synaptic pruning and hepatocyte glucose regulation. Taken together, the literature demonstrates the important and active role that HLA molecules play in transduction of intracellular signaling, mechanisms which are misappropriated in donor vascular cells upon engagement by donor-specific HLA antibodies, leading to graft injury.

Acknowledgments The authors would like to thank KAT and AR for critical review of this manuscript. This work was supported by the National Institute of Allergy and Infectious Diseases Grant RO1 AI 042819 and NIH U018I077821.

\section{Compliance with Ethics Guidelines}

Conflict of Interest Nicole M. Valenzuela and Elaine F. Reed declare that they have no conflict of interest.

Human and Animal Rights and Informed Consent This article does not contain any studies with human or animal subjects performed by any of the authors.

\section{References}

Papers of particular interest, published recently, have been highlighted as:

- Of importance

•- Of major importance

1. Frank R et al. Correlations of lymphocyte subset infiltrates with donor-specific antibodies and acute antibody-mediated rejection in endomyocardial biopsies. Cardiovasc Pathol. doi:10.1016/j. carpath.2014.11.001

2. Torres IB et al. Comparing transplant glomerulopathy in the absence of $\mathrm{C} 4 \mathrm{~d}$ deposition and donor-specific antibodies to chronic antibody-mediated rejection. Clin Transpl. 2014;28:1148-54. doi: 10.1111/ctr.12433.

3. DeNicola $\mathrm{MM}$ et al. Pathologic findings in lung allografts with anti-HLA antibodies. J Heart Lung Transplant Off Pub Int Soc Heart Trans. 2013;32:326-32. doi:10.1016/j.healun.2012.11.018.

4. Valenzuela NM et al. HLA class I antibodies trigger increased adherence of monocytes to endothelial cells by eliciting an increase in endothelial P-selectin, and depending on subclass, by engaging FcgammaRs. J Immunol. 2013;190:6635-50. doi:10. 4049/jimmunol.1201434. 
5. Valenzuela NM et al. Monocyte recruitment by HLA IgGactivated endothelium: the relationship between IgG subclass and Fc $\gamma$ RIIa polymorphisms. Am J Trans. 2015. doi:10.1111/ajt. 13174.

6.• Jane-wit D et al. Alloantibody and complement promote T cellmediated cardiac allograft vasculopathy through noncanonical nuclear factor- $\mathrm{kB}$ signaling in endothelial cells. Circulation. 2013;128:2504-16. doi:10.1161/circulationaha.113.002972. The investigators treated IFN $\gamma$-activated endothelial cells with polyclonal alloserum containing HLA (class I and class II) antibodies in the absence or presence of complement, and assessed endothelial late phase activation, including transcriptional upregulation of adhesion molecules and chemokines. They found that HLA antibodies triggered increased adhesion and activation of allogeneic CD4 $T$ cells.

7. Taflin $\mathrm{C}$ et al. Study of the allogeneic response induced by endothelial cells expressing HLA class II after lentiviral transduction. In: van Endert P, editor. In antigen processing. New York: Humana Press; 2013. p. 461-72. doi:10.1007/978-1-62703-218-6_34.

8. Wang $\mathrm{C}$ et al. Rapamycin-treated human endothelial cells preferentially activate allogeneic regulatory $\mathrm{T}$ cells. J Clin Invest. 2013;123:1677-93. doi:10.1172/JCI66204. In this study, the authors evaluated the effect of chronic mTOR inhibition of endothelial cells on their alloimmunogenicity to $T$ cells. Their results suggest that rapalogues modulate endothelial antigen presenting function.

9. Jin YP et al. RNA interference elucidates the role of focal adhesion kinase in HLA class I-mediated focal adhesion complex formation and proliferation in human endothelial cells. J Immunol. 2007; 178:7911-22

10. Lepin EJ et al. HLA class I signal transduction is dependent on Rho GTPase and ROK. Biochem Biophys Res Commun. 2004;323:213-7. doi:10.1016/j.bbrc.2004.08.082.

11. Ziegler ME et al. HLA class I-mediated stress fiber formation requires ERK $1 / 2$ activation in the absence of an increase in intracellular Ca2+ in human aortic endothelial cells. Am J Physiol Cell Physiol. 2012;303:C872-882. doi:10.1152/ajpcell.00199.2012.

12. Ziegler ME et al. Characterization of the endothelial cell cytoskeleton following HLA class I ligation. PLoS One. 2012;7:e29472. doi:10.1371/journal.pone.0029472. Ziegler et al. utilized a novel proteomic approach to describe the intracellular proteins that associate with the actin cytoskeleton after endothelial stimulation with HLA I antibodies.

13. Coupel $\mathrm{S}$ et al. RhoA activation mediates phosphatidylinositol 3 kinase-dependent proliferation of human vascular endothelial cells: an alloimmune mechanism of chronic allograft nephropathy. Clin J Am Soc Nephrol JASN. 2004;15:2429-39. doi:10.1097/01. ASN.0000138237.42675.45.

14. Jin YP et al. Ligation of HLA class I molecules on endothelial cells induces phosphorylation of Src, paxillin, and focal adhesion kinase in an actin-dependent manner. J Immunol. 2002;168:541523.

15. Li F et al. Antibody ligation of human leukocyte antigen class I molecules stimulates migration and proliferation of smooth muscle cells in a focal adhesion kinase-dependent manner. Hum Immunol. 2011;72:1150-9. doi:10.1016/j.humimm.2011.09.004.

16. Yoshizawa $\mathrm{S}$ et al. Focal adhesion kinase mediates human leukocyte histocompatibility antigen class II-induced signaling in gingival fibroblasts. J Periodontal Res. 2007;42:572-9. doi:10.1111/j. 1600-0765.2007.00985.x.

17. Murali A, Rajalingam K. Small Rho GTPases in the control of cell shape and mobility. Cell Mol Life Sci CMLS. 2014;71:1703-21. doi:10.1007/s00018-013-1519-6.

18. Wojciak-Stothard B et al. Rac and Rho play opposing roles in the regulation of hypoxia/reoxygenation-induced permeability changes in pulmonary artery endothelial cells. Am J Physiol Lung Cell
Mol Physiol. 2005;288:L749-760. doi:10.1152/ajplung.00361. 2004.

19. Jindra PT et al. MHC class I and integrin ligation induce ERK activation via an mTORC2-dependent pathway. Biochem Biophys Res Commun. 2008;369:781-7. doi:10.1016/j.bbrc. 2008.02.093.

20. Geiger B et al. Environmental sensing through focal adhesions. Nat Rev Mol Cell Biol. 2009;10:21-33. doi:10.1038/nrm2593.

21. Jaqaman K, Grinstein S. Regulation from within: the cytoskeleton in transmembrane signaling. Trends Cell Biol. 2012;22:515-26. doi:10.1016/j.tcb.2012.07.006.

22. Tojkander $\mathrm{S}$ et al. Actin stress fibers-assembly, dynamics and biological roles. J Cell Sci. 2012;125:1855-64. doi:10.1242/jcs. 098087.

23. Hordijk PL. Endothelial signalling events during leukocyte transmigration. FEBS J. 2006;273:4408-15. doi:10.1111/j.1742-4658. 2006.05440.x.

24. Jin YP et al. Everolimus inhibits anti-HLA I antibody-mediated endothelial cell signaling, migration and proliferation more potently than sirolimus. Am J Transplant. 2014;14:806-19. doi:10.1111/ ajt.12669. In this work, Jin et al. report that HLA I antibodystimulated endothelial proliferation can be abrogated by rapalogues sirolimus and everolimus. Moreover, everolimus was more potent at inhibiting mTORC2 and its target ERK, and therefore was more effective at preventing endothelial migration and proliferation compared with sirolimus, which was less efficient at inhibiting mTORC2.

25. Iwasaki $\mathrm{K}$ et al. Comparative study on signal transduction in endothelial cells after anti-a/b and human leukocyte antigen antibody reaction: implication of accommodation. Transplantation. 2012;93:390-7. doi:10.1097/TP.0b013e3182424df3.

26. Narayanan $\mathrm{K}$ et al. HLA class I antibody mediated accommodation of endothelial cells via the activation of PI3K/cAMP dependent PKA pathway. Transpl Immunol. 2006;15:187-97. doi:10. 1016/j.trim.2005.09.005.

27. Jin YP et al. Anti-HLA class I antibody-mediated activation of the $\mathrm{PI} 3 \mathrm{~K} / \mathrm{Akt}$ signaling pathway and induction of $\mathrm{Bcl}-2$ and $\mathrm{Bcl}-\mathrm{xL}$ expression in endothelial cells. Hum Immunol. 2004;65:291-302. doi:10.1016/j.humimm.2004.01.002.

28. Le Bas-Bernardet $\mathrm{S}$ et al. Vascular endothelial cells evade apoptosis triggered by human leukocyte antigen-DR ligation mediated by allospecific antibodies. Transplantation. 2004;78:1729-39.

29. Hidalgo LG et al. NK cell transcripts and NK cells in kidney biopsies from patients with donor-specific antibodies: evidence for NK cell involvement in antibody-mediated rejection. Am J Transplant. 2010;10:1812-22. doi:10.1111/j.1600-6143.2010. 03201.x.

30. Sis B et al. Endothelial gene expression in kidney transplants with alloantibody indicates antibody-mediated damage despite lack of C4d staining. Am J Transplant. 2009;9:2312-23. doi:10.1111/j. 1600-6143.2009.02761.x.

31. Loupy A et al. Outcome of subclinical antibody-mediated rejection in kidney transplant recipients with preformed donor-specific antibodies. Am J Transplant. 2009;9:2561-70. doi:10.1111/j. 1600-6143.2009.02813.x.

32. Papadimitriou JC et al. Antibody-mediated allograft rejection: morphologic spectrum and serologic correlations in surveillance and for cause biopsies. Transplantation. 2013;95:128-36. doi:10. 1097/TP.0b013e3182777f28.

33.• Wiebe $\mathrm{C}$ et al. Evolution and clinical pathologic correlations of de novo donor-specific HLA antibody post kidney transplant. Am J Transplant. 2012;12:1157-67. doi:10.1111/j.1600-6143.2012. 04013.x. Wiebe et al. propose a model of the "natural history of antibodies" in which subclinical or indolent graft injury occurs close to the time of first de novo donor specific 
antibodies appearance, while the histological and clinical manifestations of graft dysfunction occur later.

34. Everly MJ et al. Incidence and impact of de novo donor-specific alloantibody in primary renal allografts. Transplantation. 2013;95: 410-7. doi:10.1097/TP.0b013e31827d62e3.

35. Mehra MR et al. International society for heart and lung transplantation working formulation of a standardized nomenclature for cardiac allograft vasculopathy-2010. J Heart Lung Transplant Off Pub Int Soc Heart Trans. 2010;29:717-27. doi:10.1016/j. healun.2010.05.017.

36. Haas $\mathrm{M}$ et al. Banff 2013 meeting report: inclusion of c4dnegative antibody-mediated rejection and antibody-associated arterial lesions. Am J Transplant. 2014;14:272-83. doi:10.1111/ajt. 12590.

37. Lobo LJ et al. Donor-specific antibodies are associated with antibody-mediated rejection, acute cellular rejection, bronchiolitis obliterans syndrome, and cystic fibrosis after lung transplantation. J Heart Lung Transplant Off Pub Int Soc Heart Trans. 2013;32: 70-7. doi:10.1016/j.healun.2012.10.007.

38. Stewart S et al. Revision of the 1996 working formulation for the standardization of nomenclature in the diagnosis of lung rejection. J Heart Lung Transplant Off Pub Int Soc Heart Trans. 2007;26: 1229-42. doi:10.1016/j.healun.2007.10.017.

39. Atkinson $\mathrm{C}$ et al. Angiogenesis occurs within the intimal proliferation that characterizes transplant coronary artery vasculopathy. $\mathrm{J}$ Heart Lung Transplant Off Pub Int Soc Heart Trans. 2005;24:5518. doi:10.1016/j.healun.2004.03.012.

40. Libby P, Zhao DX. Allograft arteriosclerosis and immune-driven angiogenesis. Circulation. 2003;107:1237-9.

41. Tanaka H et al. Endothelial and smooth muscle cells express leukocyte adhesion molecules heterogeneously during acute rejection of rabbit cardiac allografts. Am J Pathol. 1994;144:938-51.

42. Zhao XM et al. Association of thrombospondin-1 and cardiac allograft vasculopathy in human cardiac allografts. Circulation. 2001;103:525-31.

43. Jindra PT et al. Anti-MHC class I antibody activation of proliferation and survival signaling in murine cardiac allografts. $\mathrm{J}$ Immunol. 2008;180:2214-24.

44. Jindra PT et al. HLA class I antibody-mediated endothelial cell proliferation via the mTOR pathway. J Immunol. 2008;180:235766.

45. Hirohashi $\mathrm{T}$ et al. Complement independent antibody-mediated endarteritis and transplant arteriopathy in mice. Am J Transplant. 2010;10:510-7. doi:10.1111/j.1600-6143.2009.02958.x.

46. Russell PS et al. Coronary atherosclerosis in transplanted mouse hearts. II. Importance of humoral immunity. J Immunol. 1994;152: 5135-41.

47. Lepin EJ et al. Phosphorylated S6 ribosomal protein: a novel biomarker of antibody-mediated rejection in heart allografts. Am J Transplant. 2006;6:1560-71. doi:10.1111/j.1600-6143.2006. 01355.x.

48. Li F et al. Phosphorylated S6 kinase and S6 ribosomal protein are diagnostic markers of antibody-mediated rejection in heart allografts. J Heart Lung Transplant. 2014. doi:10.1016/j.healun.2014. 09.047 .

49. Tible $\mathrm{M}$ et al. Pathologic classification of antibody-mediated rejection correlates with donor-specific antibodies and endothelial cell activation. J Heart Lung Transplant. 2013;32:769-76. doi: 10.1016/j.healun.2013.05.012. The parallel findings reported by $\mathrm{Li}$ et al. and Tible et al. demonstrate that detection of activated signaling proteins implicated in HLA I antibody induced intracellular signaling (including phosphorylated S6K and S6RP) facilitates diagnosis of antibody-mediated rejection in cardiac allografts.

50. Bruneau $\mathrm{S}$ et al. DEPTOR regulates vascular endothelial cell activation and proinflammatory and angiogenic responses. Blood.
2013;122:1833-42. doi:10.1182/blood-2013-03-488486. This study describes a novel negative regulator of $\mathrm{mTOR}$, DEPTOR, in endothelial cells and its impact on endothelial immunogenicity. Knockdown of DEPTOR resulted in constitutive activation of $m$ TOR, and increased angiogenic functions, chemokine expression and adhesion of $T$ cells, demonstrating that mTOR is a central regulator of these functions. The authors propose that DEPTOR is a putative therapeutic target to modulate mTOR activity.

51. Arora $\mathrm{S}$ et al. Effect of everolimus introduction on cardiac allograft vasculopathy-results of a randomized, multicenter trial. Transplantation. 2011;92:235-43. doi:10.1097/TP. 0b013e31822057f1.

52. Kobashigawa JA et al. Cardiac allograft vasculopathy by intravascular ultrasound in heart transplant patients: substudy from the Everolimus versus mycophenolate mofetil randomized, multicenter trial. JACC Heart Failure. 2013;1:389-99. doi:10.1016/j.jchf. 2013.07.002.

53. Daly KP et al. VEGF-C, VEGF-A and related angiogenesis factors as biomarkers of allograft vasculopathy in cardiac transplant recipients. J Heart Lung Transplant Off Pub Int Society Heart Trans. 2013;32:120-8. doi:10.1016/j.healun.2012.09.030.

54. Reinders ME et al. Expression patterns of vascular endothelial growth factor in human cardiac allografts: association with rejection. Transplantation. 2003;76:224-30. doi:10.1097/01.TP. 0000071363.55007.D0.

55. Lemstrom KB et al. Vascular endothelial growth factor enhances cardiac allograft arteriosclerosis. Circulation. 2002;105:2524-30.

56. Bieri $\mathrm{M}$ et al. Anti-HLA I antibodies induce VEGF production by endothelial cells, which increases proliferation and paracellular permeability. Int J Biochem Cell Biol. 2009;41:2422-30. doi:10. 1016/j.biocel.2009.06.009.

57. Galvani $\mathrm{S}$ et al. A key role for matrix metalloproteinases and neutral sphingomyelinase-2 in transplant vasculopathy triggered by anti-HLA antibody. Circulation. 2011;124:2725-34. doi:10. 1161/CIRCULATIONAHA.111.021790.

58. Fishbein GA, Fishbein MC. Morphologic and immunohistochemical findings in antibody-mediated rejection of the cardiac allograft. Hum Immunol. 2012;73:1213-7. doi:10.1016/j.humimm. 2012.07.011.

59. Tinckam KJ et al. Glomerular monocytes predict worse outcomes after acute renal allograft rejection independent of $\mathrm{C} 4 \mathrm{~d}$ status. Kidney Int. 2005;68:1866-74. doi:10.1111/j.1523-1755.2005. 00606.x.

60. Xu L et al. Increased macrophage density of cardiac allograft biopsies is associated with antibody-mediated rejection and alloantibodies to HLA antigens. Clin Transpl. 2014;28:554-60. doi: $10.1111 /$ ctr. 12348 .

61. Hirohashi $\mathrm{T}$ et al. A novel pathway of chronic allograft rejection mediated by NK cells and alloantibody. Am J Transplant. 2012;12:313-21. doi:10.1111/j.1600-6143.2011.03836.x.

62. Valenzuela NM et al. Blockade of p-selectin is sufficient to reduce MHC I antibody-elicited monocyte recruitment in vitro and in vivo. Am J Transplant. 2013;13:299-311. doi:10.1111/ajt. 12016.

63. Abe $\mathrm{T}$ et al. Graft-derived CCL2 increases graft injury during antibody-mediated rejection of cardiac allografts. Am J Transplant. 2014;14:1753-64. doi:10.1111/ajt.12780.

64. Lowenstein CJ et al. Regulation of Weibel-Palade body exocytosis. Trends Cardiovasc Med. 2005;15:302-8. doi:10.1016/j.tcm. 2005.09.005.

65. Yamakuchi $\mathrm{M}$ et al. Antibody to human leukocyte antigen triggers endothelial exocytosis. Proc Natl Acad Sci U S A. 2007;104: 1301-6. doi:10.1073/pnas.0602035104.

66. Morrell $\mathrm{CN}$ et al. In vivo platelet-endothelial cell interactions in response to major histocompatibility complex alloantibody. Circ 
Res. 2008;102:777-85. doi:10.1161/CIRCRESAHA.107. 170332.

67. Kuo $\mathrm{HH}$ et al. Platelets in early antibody-mediated rejection of renal transplants. Clin J Am Soc Nephrol JASN. 2014. doi:10. 1681/ASN.2013121289.

68. da Costa Martins P et al. Platelet-monocyte complexes support monocyte adhesion to endothelium by enhancing secondary tethering and cluster formation. Arterioscler Thromb Vasc Biol. 2004;24:193-9. doi:10.1161/01.ATV.0000106320.40933.E5.

69. Mannam VK et al. The fate of renal allografts hinges on responses of the microvascular endothelium. Exp Mol Pathol. 2013;94:398411. doi:10.1016/j.yexmp.2012.06.002.

70. Naemi FM et al. Anti-donor HLA class I antibodies: pathways to endothelial cell activation and cell-mediated allograft rejection. Transplantation. 2013;96:258-66. doi:10.1097/TP. 0b013e3182985504.

71. Meguro M et al. Ligation of IFN-gamma-induced HLA-DR molecules on fibroblasts induces RANTES expression via c-Jun Nterminal kinase (JNK) pathway. Cytokine. 2003;22:107-15.

72. Wang $\mathrm{C}$ et al. Rapamycin antagonizes TNF induction of VCAM-1 on endothelial cells by inhibiting mTORC2. Isr J Exp Med. 2014;211:395-404. doi:10.1084/jem.20131125.

73. Samsonov D et al. Differential activation of human $\mathrm{T}$ cells to allogeneic endothelial cells, epithelial cells and fibroblasts in vitro. Transl Res. 2012;1:4. doi:10.1186/2047-1440-1-4.

74. Shiao SL et al. Human effector memory CD4+ T cells directly recognize allogeneic endothelial cells in vitro and in vivo. $\mathrm{J}$ Immunol. 2007;179:4397-404.

75. Ma W, Pober JS. Human endothelial cells effectively costimulate cytokine production by, but not differentiation of, naive CD4+ T cells. J Immunol. 1998;161:2158-67.

76. Kambayashi T, Laufer TM. Atypical MHC class II-expressing antigen-presenting cells: can anything replace a dendritic cell? Nat Rev Immunol. 2014;14:719-30. doi:10.1038/nri3754.

77. Pober JS, Tellides G. Participation of blood vessel cells in human adaptive immune responses. Trends Immunol. 2012;33:49-57. doi:10.1016/j.it.2011.09.006.

78. Taflin $\mathrm{C}$ et al. Human endothelial cells generate Th17 and regulatory T cells under inflammatory conditions. Proc Natl Acad Sci U S A. 2011;108:2891-6. doi:10.1073/pnas.1011811108.

79. Gaughan A et al. Key role for CD4 T cells during mixed antibodymediated rejection of renal allografts. Am J Transplant. 2014;14: 284-94. doi:10.1111/ajt.12596.

80. Sumen $\mathrm{C}$ et al. T cell receptor antagonism interferes with MHC clustering and integrin patterning during immunological synapse formation. J Cell Biol. 2004;166:579-90. doi:10.1083/jcb. 200404059.

81. Vogt $\mathrm{AB}$ et al. Clustering of MHC-peptide complexes prior to their engagement in the immunological synapse: lipid raft and tetraspan microdomains. Immunol Rev. 2002;189:136-51.

82. Miro F et al. T cell-dependent activation of dendritic cells requires IL-12 and IFN-gamma signaling in T cells. J Immunol. 2006; 177: 3625-34.

83. Puccetti P, Grohmann U. IDO and regulatory T cells: a role for reverse signalling and non-canonical NF-kappaB activation. Nat Rev Immunol. 2007;7:817-23. doi:10.1038/nri2163.

84. Al-Alwan MM et al. Cutting edge: dendritic cell actin cytoskeletal polarization during immunological synapse formation is highly antigen-dependent. J Immunol. 2003;171:4479-83.

85. Delaguillaumie A et al. Contrasting cytoskeletal regulation of MHC class II peptide presentation by human B cells or dendritic cells. Eur J Immunol. 2008;38:1096-105. doi:10.1002/eji. 200737455.

86. Drénou B et al. MHC class II signaling function is regulated during maturation of plasmacytoid dendritic cells. J Leukoc Biol. 2005;77:560-7. doi:10.1189/jlb.0704423.
87. Bains SK et al. Mitochondria control of cell death induced by antiHLA-DR antibodies. Leukemia. 2003;17:1357-65. doi:10.1038/ sj.leu.2402976.

88. Holling TM et al. Function and regulation of MHC class II molecules in T-lymphocytes: of mice and men. Hum Immunol. 2004;65:282-90. doi:10.1016/j.humimm.2004.01.005.

89. Matsuoka $\mathrm{T}$ et al. Monocytes are differentially activated through HLA-DR, -DQ, and -DP molecules via mitogen-activated protein kinases. J Immunol. 2001;166:2202-8.

90. Nabavi N et al. Signalling through the MHC class II cytoplasmic domain is required for antigen presentation and induces B7 expression. Nature. 1992;360:266-8. doi:10.1038/360266a0.

91. Setterblad N et al. Cognate MHC-TCR interaction leads to apoptosis of antigen-presenting cells. J Leukoc Biol. 2004;75:103644. doi:10.1189/jlb.0703356.

92. Truman JP et al. HLA class II molecule signal transduction leads to either apoptosis or activation via two different pathways. Cell Immunol. 1996;172:149-57. doi:10.1006/cimm.1996.0227.

93. Altomonte $\mathrm{M}$ et al. The overlooked "nonclassical" functions of major histocompatibility complex (MHC) class II antigens in immune and nonimmune cells. J Cell Physiol. 1999;179:251-6. doi: 10.1002/(SICI)1097-4652(199906)179:3<251::AID-JCP2>3.0. $\mathrm{CO} ; 2-\mathrm{P}$.

94. Faure-Andre $\mathrm{G}$ et al. Regulation of dendritic cell migration by CD74, the MHC class II-associated invariant chain. Science. 2008;322:1705-10. doi:10.1126/science.1159894.

95. Rubio $\mathrm{G}$ et al. Cross-linking of MHC class I molecules on human NK cells inhibits NK cell function, segregates MHC I from the NK cell synapse, and induces intracellular phosphotyrosines. J Leukoc Biol. 2004;76:116-24. doi:10.1189/jlb.1103597.

96. Corral-San Miguel R et al. MHC-I molecules selectively inhibit cell-mediated cytotoxicity triggered by ITAM-coupled activating receptors and 2B4. PLoS ONE. 2014;9:e107054. doi:10.1371/ journal.pone. 0107054 . In this work, NK cell cytotoxicity against tumor cells was significantly inhibited by concurrent crosslinking of MHC I, which induced negative crosstalk signaling pathways to antagonize activating receptors.

97. El Fakhry Y et al. Delineation of the HLA-DR region and the residues involved in the association with the cytoskeleton. J Biol Chem. 2004;279:18472-80. doi:10.1074/jbc.M401159200.

98. Zhang $X$ et al. HLA class I molecules partner with integrin $\beta 4$ to stimulate endothelial cell proliferation and migration. Sci Signal. 2010;3:ra85-10.1126/scisignal.2001158.

99. Capps GG, Zuniga MC. Phosphorylation of class I MHC molecules in the absence of phorbol esters is an intracellular event and may be characteristic of trafficking molecules. Mol Immunol. 2000;37:59-71.

100. Guild BC, Strominger JL. HLA-A2 antigen phosphorylation in vitro by cyclic AMP-dependent protein kinase. Sites of phosphorylation and segmentation in class I major histocompatibility complex gene structure. J Biol Chem. 1984;259:13504-10.

101. Xu S et al. Constitutive MHC class I molecules negatively regulate TLR-triggered inflammatory responses via the Fps-SHP-2 pathway. Nat Immunol. 2012;13:551-9. doi:10.1038/ni.2283.

102. Anderson HA, Roche PA. Phosphorylation regulates the delivery of MHC class II invariant chain complexes to antigen processing compartments. J Immunol. 1998;160:4850-8.

103. Engering A, Pieters J. Association of distinct tetraspanins with MHC class II molecules at different subcellular locations in human immature dendritic cells. Int Immunol. 2001;13:127-34.

104. Hoorn Tet al. Dynamics within tetraspanin pairs affect MHC class II expression. J Cell Sci. 2012;125:328-39. doi:10.1242/jcs. 088047.

105. Lagaudriere-Gesbert $\mathrm{C}$ et al. The tetraspanin protein CD82 associates with both free HLA class I heavy chain and heterodimeric beta 2-microglobulin complexes. J Immunol. 1997;158:2790-7. 
106. Petersen $\mathrm{SH}$ et al. The role of tetraspanin CD63 in antigen presentation via MHC class II. Eur J Immunol. 2011;41:2556-61. doi:10. 1002/eji.201141438.

107. Unternaehrer JJ et al. The tetraspanin CD9 mediates lateral association of MHC class II molecules on the dendritic cell surface. Proc Natl Acad Sci U S A. 2007;104:234-9. doi:10.1073/pnas. 0609665104

108. Mori $\mathrm{Y}$ et al. Inhibitory immunoglobulin-like receptors LILRB and PIR-B negatively regulate osteoclast development. J Immunol. 2008;181:4742-51.

109. Shiroishi M et al. Structural basis for recognition of the nonclassical MHC molecule HLA-G by the leukocyte Ig-like receptor B2 (LILRB2/LIR2/ILT4/CD85d). Proc Natl Acad Sci U S A. 2006;103:16412-7. doi:10.1073/pnas.0605228103.

110. Willcox BE et al. Crystal structure of HLA-A2 bound to LIR-1, a host and viral major histocompatibility complex receptor. Nat Immunol. 2003;4:913-9. doi:10.1038/ni961.

111. Jones DC et al. HLA class I allelic sequence and conformation regulate leukocyte Ig-like receptor binding. J Immunol. 2011;186: 2990-7. doi:10.4049/jimmunol.1003078.

112. Held W, Mariuzza RA. Cis interactions of immunoreceptors with MHC and non-MHC ligands. Nat Rev Immunol. 2008;8:269-78. doi:10.1038/nri2278.

113. Liu X et al. Intracellular MHC class II molecules promote TLRtriggered innate immune responses by maintaining activation of the kinase Btk. Nat Immunol. 2011;12:416-24. doi:10.1038/ni. 2015.

114.• Lee H et al. Synapse elimination and learning rules co-regulated by MHC class I H2-Db. Nature. 2014;509:195-200. doi:10.1038/ nature13154. New work in murine models shows that MHC I is required for neuronal patterning. Knockdown of classical MHC I prevents synapse elimination and neuronal pruning that is essential for development in the central nervous system, identifying a novel role for MHC I outside the classical functions in the immune system.

115. Ramalingam TS et al. Interaction of class I human leukocyte antigen (HLA-I) molecules with insulin receptors and its effect on the insulin-signaling cascade. Mol Biol Cell. 1997;8:2463-74.

116. Reiland J, Edidin M. Chemical cross-linking detects association of insulin receptors with four different class I human leukocyte antigen molecules on cell surfaces. Diabetes. 1993;42:619-25.

117. Dixon-Salazar TJ et al. MHC class I limits hippocampal synapse density by inhibiting neuronal insulin receptor signaling. J Neurosci Off J Soc Neurosci. 2014;34:11844-56. doi:10.1523/ JNEUROSCI.4642-12.2014.

118. Giancotti FG. Targeting integrin beta4 for cancer and antiangiogenic therapy. Trends Pharmacol Sci. 2007;28:506-11. doi: 10.1016/j.tips.2007.08.004.

119. Zhang X, Reed EF. HLA class I: an unexpected role in integrin beta4 signaling in endothelial cells. Hum Immunol. 2012;73: 1239-44. doi:10.1016/j.humimm.2012.06.013. 